\title{
DINÁMICA DE CRECIMIÉNTO DE STEVIA CULTIVADA EN SUSTRATOS ORGÁNICOS EN INVERNADERO
}

\author{
GROWTH DYNAMICS OF STEVIA CULTIVATED IN \\ ORGANIC SUBSTRATES IN GREENHOUSE
}

\section{José C. Romero-Figueroa', Ma. de las Nieves Rodríguez-Mendoza ${ }^{1}$, J. Alberto S. Escalante-Estrada', Ma. del Carmen Gutiérrez-Castorena', Cecilia B. Peña-Valdivia', José A. Cueto-Wong ${ }^{2}$ y Esteban Burguete-Hernández ${ }^{1}$}

\begin{abstract}
'Campus Montecillo, Colegio de Postgraduados. km 36.5 Carretera México-Texcoco. 56230, Montecillo, Texcoco, Estado de México. ${ }^{2}$ Centro Nacional de Investigación Disciplinaria en Relación Agua, Suelo, Planta, Atmósfera. km 6.5 margen derecha canal Sacramento. 35140, Gómez Palacio, Durango, México.
\end{abstract}

*Autor para correspondencia (marinie@colpos.mx)

\section{RESUMEN}

En México existen muchas especies de Stevia; sin embargo, es poca la información sobre el crecimiento y desarrollo de éstas. Se evaluaron en invernadero la dinámica e índices de crecimiento de S. pilosa Lag. y S. tomentosa H.B.K. cultivadas en sustrato con diferentes proporciones de fibra de coco:vermicompost (0:100, 25:75, 50:50, 75:25 y 100:0) con base en volumen. La dinámica y la tasa de crecimiento se midieron seis veces entre los 42 y 82 días después de la emergencia. Los resultados indicaron que hay diferencias en las variables estudiadas con respecto a la cantidad de vermicompost. En la distribución de biomasa, los tallos de plantas de S. pilosa y S. tomentosa con el $100 \%$ de vermicompost asignaron el 54.7 y $48.1 \%$, respectivamente, mientras que sin vermicompost la asignación fue 38.5 y $23.2 \%$, respectivamente. Las plantas de S. tomentosa con 75 y $100 \%$ de vermicompost desarrollaron la mayor área foliar (440.87 y 426.98 $\mathrm{cm}^{2}$, respectivamente) en el muestreo final. La tasa relativa de crecimiento fue mayor en el primer muestreo en ambas especies; en S. pilosa se obtuvo $0.954 \mathrm{~g} \mathrm{~g} \mathrm{~g}^{-1} \mathrm{~d}^{-1}$ (50\% vermicompost) y en $\mathrm{S}$. tomentosa $0.657 \mathrm{~g} \mathrm{~g}^{-1} \mathrm{~d}^{-1}(25 \% \mathrm{de}$ vermicompost). Las mejores mezclas fueron las que contenían 75 y $100 \%$ de vermicompost, lo que significa que las plantas prefieren materiales orgánicos altamente humificados, tal como ocurre en condiciones naturales. S. pilosa fue la especie con mejor dinámica de crecimiento e índices de eficiencia.

Palabras clave: Stevia pilosa, Stevia tomentosa, área foliar, índices de crecimiento, vermicompost.

\section{SUMMARY}

In Mexico there are many species of Stevia; however, there is little information on their growth and development. The dynamics and growth rates of plants of S. pilosa Lag. and S. tomentosa H.B.K. were evaluated in greenhouse grown on substrate with different proportions of coconut fiber:vermicompost $(0: 100$, $25: 75,50: 50,75: 25$ and 100:0) based on volume. Dynamics and growth rates were evaluated six times between 42 and 82 days after seedling emergence. Results indicated that there were differences in the studied variables in relation to the amount of vermicompost. In biomass distribution, the stems of $S$. pilosa and S. tomentosa with $100 \%$ of vermicompost assigned 54.7 and $48.1 \%$, respectively, while without vermicompost the assignation was 38.5 and 23.2 $\%$, respectively. Plants of $S$. tomentosa with 75 and $100 \%$ of vermicompost developed the largest leaf area (440.87 and $426.98 \mathrm{~cm}^{2}$, respectively) at the final sampling. The relative growth rate was higher at the first sampling in both species; it was $0.954 \mathrm{~g} \mathrm{~g}^{-1} \mathrm{~d}^{-1}$ (50\% vermicompost) in S. pilosa and 0.657 $\mathrm{g} \mathrm{g}^{-1} \mathrm{~d}^{-1}$ (25\% vermicompost) in S. tomentosa. The best mixtures were those with 75 and $100 \%$ of vermicompost, which means that plants prefer highly humified organic materials, as it occurs in natural conditions. S. pilosa was the species with the best growth dynamics and efficiency indices.

Index words: Stevia pilosa, Stevia tomentosa, leaf area, growth indices, vermicompost.

\section{INTRODUCCIÓN}

El género Stevia, que pertenece a la tribu Eupatorieae, familia Asteraceae, se extiende desde el suroeste de los Estados Unidos de América hasta el norte de Argentina. Se estima que al género Stevia lo integran 300 especies, de las cuales más de 80 se encuentran en Norteamérica, y de éstas 70 son nativas de México (Grashoff, 1972; Com pers. $)^{1}$. El $80 \%$ de las especies de Stevia son analizadas con fines fitoquímicos (Cerda-García-Rojas y Pereda-Miranda, 2001; Hernández et al., 1998).

En México, algunas especies se utilizan para ornato en el día tradicional de muertos por los colores característicos de las flores (Villavicencio y Pérez, 1995) y en la herbolaria por su potencial farmacéutico (Cariño-Cortes et al., 2002; Com. pers²; Molina-Mendoza et al., 2012). Investigaciones recientes señalan la importancia de S. pilosa y S. tomentosa como fitofármaco o complemento alimenticio quimiopreventivo.

Calderón y Rzedowski (2005) reportaron que S. pilosa y S. tomentosa se localizan en altitudes entre 2300 y 4000

'Grashoff J. L. (1972) A Systematic Study of the North and Central American Species of Stevia. PhD Dissertation. University of Texas. Austin, Texas. 609 p.

${ }^{2}$ Cariño-Cortes R., M. Arriaga-Alba, A. Hernández-Ceruelos, R. Álvarez-García y M. González-Avila. (2002) Actividad antimutagénica, antioxidante y antiinflamatoria de Stevia pilosa y S. eupatoria, una alternativa terapéutica. XI Congreso Nacional de Biotecnología y Bioingeniería. Sociedad Mexicana de Biotecnología y Bioingeniería. Mérida, Yucatán. p. 19. 
$\mathrm{m}$, en lugares donde llueve de 700 a $1200 \mathrm{~mm}$ anuales, condiciones que se correlacionan con bosques de Pinus y Quercus y donde se acumula materia orgánica en el horizonte superficial con diferentes grados de descomposición.

No obstante, la importancia de las especies de Stevia existe poca información sobre su crecimiento. El análisis del crecimiento puede ser un método cuantitativo que permita describir todo el sistema de la planta en condiciones controladas y generar conocimiento sobre su desarrollo en condiciones ex situ (Azarpour et al., 2014); además, estos estudios son una alternativa para explicar diferencias en el crecimiento debidas a su constitución genética o al ambiente, para evaluar la distribución de la biomasa vegetal y calcular índices de eficiencia del crecimiento como la tasa relativa de crecimiento (TRC), la tasa absoluta de crecimiento (TAC) y la tasa de asimilación neta (TAN) (Hunt, 2017; Prusinkiewicz, 2004). La TAN permite cuantificar la fotosíntesis laminar y su relación con la producción de materia seca elaborada por la planta, determinada fundamentalmente por la fotosíntesis y la respiración. La TRC permite realizar comparaciones equitativas, y normalmente relaciona una parte de la planta con la materia seca total. El área foliar específica (AFE) es un índice morfológico que describe la proporción follaje entre el potencial fotosintético y el respiratorio; es decir, la relación entre el área foliar total y la materia seca total de la planta (Hunt, 2017).

El vermicompost y la fibra de coco (Cocos nucifera) son materiales orgánicos que pueden simular las condiciones edáficas comunes en los suelos de bosque. Por lo tanto, el objetivo de la presente investigación fue evaluar la dinámica y los índices de crecimiento de plantas de S. pilosa y $S$. tomentosa en función del porcentaje de vermicompost como sustrato humificado para su crecimiento bajo condiciones de invernadero. La hipótesis es que diferentes grados de descomposición y relaciones de fibra de coco y vermicompost proporcionan condiciones ambientales que influyen de manera particular en el desarrollo de S. pilosa y S. tomentosa.

\section{MATERIALES Y MÉTODOS}

\section{Material vegetal y experimento en invernadero}

Aquenios, provenientes de El Hiloche, estado de Hidalgo, fueron desinfectados con hipoclorito de sodio al $5 \%$ y Microdyn ${ }^{\circledR}$ por 10 min y se colocaron en agua por $20 \mathrm{~min}$ para favorecer la germinación. Posteriormente, se sembraron cinco aquenios por alvéolo (con capacidad de 85.2 $\mathrm{mL}$ ) en charolas de plástico con 50 cavidades, de acuerdo con el protocolo reportado por Sagakuchi y Kan (1982). El sustrato utilizado para almácigos fue una mezcla de tur- ba (PRO-MIX® FLEX) y vermiculita (Agrolita $\left.{ }^{\circledR}\right)(70: 30, v: v)$. Después de la emergencia (16 días después de la siembra) se aplicó riego con solución nutritiva Universal Steiner con 25 \% de concentración (Steiner, 1984). Por la heterogeneidad de las poblaciones de Stevia se seleccionaron plantas vigorosas para colocarlas en vasos de poliestireno de 250 $\mathrm{mL}$ con el mismo tipo de sustrato (una planta por vaso). Después de 12 días se trasplantaron en vasos de poliestireno de $1 \mathrm{~L}$ con los tratamientos establecidos en la investigación.

\section{Sustratos utilizados}

La fibra de coco marca AQTEX® y el vermicompost $(\mathrm{pH}$ 8.5, MO (materia orgánica) $19.56 \%$, CIC (capacidad de intercambio catiónico) $25.2 \mathrm{cmol}^{(+)} \mathrm{kg}^{-1}, \mathrm{CE}$ (conductividad eléctrica) $6.75 \mathrm{dS} \mathrm{m}^{-1}, \mathrm{~N} 1.47 \%$ y P, K, Ca, Mg y Na con 23, 5939, 900, 73 y $1283 \mathrm{mg} \mathrm{kg}^{-1}$, respectivamente).

\section{Diseño experimental y arreglo de tratamientos}

El diseño experimental fue completamente al azar con un diseño de tratamiento factorial $(2 \times 5)$; el primer factor corresponde a las especies de Stevia y el segundo factor a las mezclas de los sustratos, que corresponden a proporciones (volumen:volumen) de fibra de coco:vermicompost (0:100, $25: 75,50: 50,75: 25$ y 100:0 \%) lo que genera un total de 10 tratamientos. Se establecieron 25 repeticiones de cada tratamiento y las unidades experimentales fueron plantas individuales colocadas en bancales en el invernadero.

\section{Condiciones de crecimiento}

El riego fue con agua del grifo ( $\mathrm{pH} 7.6$ y CE $\left.0.45 \mathrm{dS} \mathrm{m}^{-1}\right)$ previamente acidulada ( $\mathrm{pH}$ 5.5). Para el control de la temperatura se colocó malla sombra negra (40\%). Las condiciones promedio fueron: temperatura máxima de $38.4{ }^{\circ} \mathrm{C}$, mínima de $9.8^{\circ} \mathrm{C}$, humedad relativa de $78.8 \%$, densidad de flujo de fotones fotosintéticos interna de 590 y externa de $2273 \mu_{\mathrm{mol} \mathrm{s}}^{-1} \mathrm{~m}^{-2}$.

\section{Análisis de crecimiento}

Para evaluar el crecimiento se realizaron muestreos destructivos a los $42,50,58,66,74$ y 82 días después de la emergencia (dde), tomando al azar cinco plantas por tratamiento. Las variables evaluadas fueron altura de la planta (cm) de la base al ápice del tallo, longitud radical a partir de la base del tallo hasta el ápice radical (cm) y el diámetro del tallo (mm). La planta se diseccionó en hojas, tallo y raíz; el área foliar (AF) se determinó con la metodología propuesta por Rincón et al. (2012) con una cámara fotográfica marca Samsung Semi-Profesional modelo WB1 100F (Seúl, Corea del Sur); posteriormente se secó el material vegetal en una 
estufa modelo BLUE M (Blue Island, Illinois, USA) con circulación de aire a $47{ }^{\circ} \mathrm{C}$ durante $72 \mathrm{~h}$. Para cuantificar la materia seca se pesaron los órganos en una balanza analítica modelo PW254 (ADAM EQUIPMENTß). Se determinó el número de hojas (NH) por planta y los índices de eficiencia (Hunt, 1982) se calcularon con los datos de peso seco y AF. También se calculó la TRC $=\ln P S_{2}-\ln P S_{1} / t_{2}-t_{1}$, donde: In = logaritmo natural; el área foliar específica (AFE = AF/ $\mathrm{PSH})$; la razón de área foliar (RAF = AF/PSP) y la relación peso foliar (RPF $=$ PSH/PSP). Las abreviaturas utilizadas son TRC: tasa relativa de crecimiento, PS: : peso seco en el tiempo 1, PS : peso seco en el tiempo 2, $t_{1}$ : tiempo 1 (días), $\mathrm{t}_{2}$ : tiempo 2 (días), PSH : peso seco de hojas, PSP. peso seco de planta, PST: peso seco de tallo, PSR: peso seco de raíz.

\section{Análisis estadístico}

El análisis de componentes principales (ACP) a partir del segundo muestreo, utilizando el programa SAS Institute, (2015). El criterio para seleccionar las variables dentro de los componentes principales fue que obtuvieran el valor más cercano a 1. Después se llevó a cabo un análisis de varianza y pruebas de comparación de medias (Duncan a= 0.05) con el programa SAS.

Con las variables seleccionadas en los componentes principales y con el programa Curve Expert Professional versión 1.2.3. 2010-2011 (Daniel G. Hyams ${ }^{\circledR}$ ) se hicieron ajustes a funciones polinomiales con base en el mayor coeficiente de determinación $\left(R^{2}\right)$ y el menor error estándar.

\section{RESULTADOS Y DISCUSIÓN}

\section{Análisis de componentes principales}

Los resultados que se muestran en la presente investigación son interpretados con base en los valores y vectores propios (Afifi et al., 2012). Los tres primeros componentes explicaron el $83.7 \%$ de la variación total. La matriz de correlaciones generada a partir del ACP mostró que 46 valores (50.5\%) fueron estadísticamente significativos, lo cual implica una alta correlación entre las características evaluadas (Siswadi et al., 2012), con variables de mayor y menor participación, lo que puede deberse principalmente a diferencias entre especies y a la proporción de vermicompost al que estuvieron expuestas.

Con el ACP se establecieron grupos de comportamiento fisiológico y de crecimiento en las plantas. El NH, AF, PSH, PST y PSR fueron las características con mayor valor descriptivo del componente principal 1 denominado "componente de crecimiento" (Cuadro 1), que representa el incremento en biomasa y número de los diferentes órganos de la planta (hojas, tallo y raíz).

Los valores significativos en el componente principal 2 "eficiencia y potencial fotosintético" de las plantas para producir nuevo tejido, fueron TRC, AFE y RAF (Cuadro 1). En el componente principal 3 "masa foliar" sólo fue significativa la RPF, que indica el desarrollo foliar de las plantas en función de la proporción de vermicompost utilizada para el crecimiento de la planta.

En el análisis de varianza se detectaron diferencias significativas $(P \leq 0.05)$ en la mayoría de las variables de mayor influencia en la definición de los componentes principales para las fuentes de variación. Las dos especies de Stevia tuvieron diferentes respuestas en función de la proporción de vermicompost; sin embargo, la interacción entre especie y sustrato no tuvo efecto significativo sobre la tasa relativa de crecimiento (Cuadro 2); esto indica que factores como agua, luz, nutrimentos, microorganismos no modificaron el peso seco.

Con base en la prueba de Duncan $(P \leq 0.05)$ se encontraron diferencias estadísticas entre los tratamientos. El

Cuadro 1. Vectores propios para las variables respuesta de dos especies de Stevia en función de la proporción de vermicompost.

\begin{tabular}{|c|c|c|c|}
\hline Variables & $\begin{array}{l}\text { Componente } \\
\text { de crecimiento }\end{array}$ & $\begin{array}{c}\text { Eficiencia } \\
\text { fotosintética }\end{array}$ & Masa foliar \\
\hline$\overline{A L T}(\mathrm{~cm})$ & 0.30 & 0.06 & -0.33 \\
\hline LR (cm) & 0.26 & -0.28 & -0.12 \\
\hline $\mathrm{NH}$ & $0.34 \S$ & 0.11 & 0.05 \\
\hline DIA (mm) & 0.29 & 0.16 & -0.23 \\
\hline $\mathrm{AF}\left(\mathrm{cm}^{2}\right)$ & $0.33 \S$ & 0.18 & 0.05 \\
\hline PSH (g) & $0.32^{\S}$ & 0.06 & 0.27 \\
\hline PST (g) & $0.33 \S$ & 0.08 & -0.02 \\
\hline PSR (g) & $0.33 \S$ & -0.09 & -0.03 \\
\hline $\operatorname{TRC}\left(\mathrm{g} \mathrm{g}^{-1} \mathrm{~d}^{-1}\right)$ & -0.14 & 0.478 & 0.19 \\
\hline $\operatorname{TAC}\left(\mathrm{g} \mathrm{d}^{-1}\right)$ & 0.30 & 0.20 & 0.21 \\
\hline $\operatorname{TAN}\left(\mathrm{g} \mathrm{cm}^{-2} \mathrm{~d}^{-1}\right)$ & 0.25 & 0.21 & 0.22 \\
\hline $\operatorname{AFE}\left(\mathrm{cm}^{2} \mathrm{~g}^{-1}\right)$ & -0.14 & $0.55 \S$ & -0.21 \\
\hline $\operatorname{RAF}\left(\mathrm{cm}^{2} \mathrm{~g}^{-1}\right)$ & -0.12 & $0.45 \S$ & -0.37 \\
\hline $\operatorname{RPF}\left(\mathrm{g} \mathrm{g}^{-1}\right)$ & -0.10 & 0.14 & $0.66^{\S}$ \\
\hline
\end{tabular}

sCoeficientes con mayor valor absoluto para describir al componente principal. ALT: altura de la planta, LR: longitud radical, $\mathrm{NH}$ : número de hojas, DIA: diámetro del tallo, AF: área foliar, PSH: peso seco de hojas, PST: peso seco de tallos, PSR: peso seco de raíz, TRC: tasa relativa de crecimiento, TAC: tasa absoluta de crecimiento, TAN: tasa de asimilación neta, AFE: área foliar específica, RAF: razón de área foliar, RPF: relación peso foliar. 
Cuadro 2. Significancia estadística para las variables con mayor peso en tres componentes principales en la producción de Stevia en función de la proporción de vermicompost.

\begin{tabular}{|c|c|c|c|c|c|c|c|c|c|}
\hline \multirow{3}{*}{ Fuente de variación } & \multicolumn{5}{|c|}{ Componente del crecimiento } & \multicolumn{3}{|c|}{ Eficiencia fotosintética } & \multirow{2}{*}{$\begin{array}{c}\text { Masa foliar } \\
\text { RPF }\end{array}$} \\
\hline & $\mathrm{Al}$ & AF & PSH & PST & PSR & TRC & AFE & RAF & \\
\hline & $\mathrm{NH}$ & $\mathrm{cm}^{2}$ & g & g & g & $\mathrm{g} \mathrm{g}^{-1} \mathrm{~d}^{-1}$ & $\mathrm{~cm}^{2} \mathrm{~g}^{-1}$ & $\mathrm{~cm}^{2} \mathrm{~g}^{-1}$ & $\mathrm{~g} \mathrm{~g}^{-1}$ \\
\hline Especie & $\star \star *$ & $\star \star$ & ** & $\star \star$ & $\star \star$ & * & $\star \star$ & ** & $\star \star$ \\
\hline Sustrato & $\star *$ & $\star \star$ & ** & $\star \star$ & $\star \star$ & $\star \star$ & ** & $\star *$ & $\star \star$ \\
\hline Especie $\times$ sustrato & $\star *$ & $\star *$ & ** & ** & $\star \star$ & ns & ** & ** & $\star \star *$ \\
\hline
\end{tabular}

*, $\star \star$ Significancia estadística a una probabilidad de 0.05 y 0.01, respectivamente. ns: no significativo, NH: número de hojas, AF: área foliar, PSH: peso seco de hojas, PST: peso seco de tallos, PSR: peso seco de raíz, TRC: tasa relativa de crecimiento, AFE: área foliar específica, RAF: razón de área foliar, RPF: relación peso foliar.

Cuadro 3. Medias de las variables con mayor peso de los primeros tres componentes principales en función de los tratamientos.

\begin{tabular}{|c|c|c|c|c|c|c|c|c|c|}
\hline \multirow{3}{*}{$\begin{array}{l}\text { Especie (porcentaje de } \\
\text { vermicompost) }\end{array}$} & \multicolumn{5}{|c|}{ Componente del crecimiento } & \multicolumn{3}{|c|}{ Eficiencia fotosintética } & \multirow{2}{*}{$\begin{array}{c}\text { Masa } \\
\text { foliar } \\
\text { RPF }\end{array}$} \\
\hline & \multirow{2}{*}{$\mathrm{NH}$} & AF & PSH & PST & PSR & TRC & AFE & RAF & \\
\hline & & $\mathrm{cm}^{2}$ & g & g & g & $\mathrm{g} \mathrm{g}^{-1} \mathrm{~d}^{-1}$ & $\mathrm{~cm}^{2} \mathrm{~g}^{-1}$ & $\mathrm{~cm}^{2} \mathrm{~g}^{-1}$ & $\mathrm{~g} \mathrm{~g}^{-1}$ \\
\hline S. pilosa (100\%) & $311.8 \mathrm{a}^{\S}$ & $270.3 a$ & $0.97 \mathrm{c}$ & $2.04 \mathrm{a}$ & $0.87 b$ & $0.33 a b$ & 397.05 a & $329.12 \mathrm{a}$ & $0.30 \mathrm{~d}$ \\
\hline S. pilosa (75\%) & $272.7 \mathrm{a}$ & $237.5 b$ & $0.92 \mathrm{c}$ & $1.85 \mathrm{ab}$ & $1.08 \mathrm{a}$ & $0.31 \mathrm{abc}$ & $340.54 b$ & $280.59 b$ & $0.26 \mathrm{e}$ \\
\hline S. pilosa (50 \%) & $222.6 b$ & $165.6 c$ & $0.81 \mathrm{c}$ & $1.38 \mathrm{c}$ & $1.04 \mathrm{a}$ & $0.30 a b c$ & $290.59 \mathrm{~cd}$ & $222.48 \mathrm{c}$ & $0.26 \mathrm{e}$ \\
\hline S. pilosa (25\%) & $101.8 \mathrm{~cd}$ & 63.6 de & 0.34 e & $0.60 d$ & $0.75 b$ & $0.24 \mathrm{~cd}$ & 267.06 cde & $205.24 \mathrm{~cd}$ & $0.22 f$ \\
\hline S. pilosa (0 \%) & $63.8 \mathrm{de}$ & $41.6 \mathrm{e}$ & $0.17 \mathrm{e}$ & $0.31 \mathrm{de}$ & $0.41 \mathrm{c}$ & $0.20 \mathrm{~d}$ & 303.21 bc & $248.74 \mathrm{bc}$ & $0.22 f$ \\
\hline S. tomentosa (100 \%) & $270.3 \mathrm{a}$ & $236.6 b$ & $1.59 \mathrm{a}$ & $1.65 \mathrm{bc}$ & $0.88 b$ & $0.36 a$ & $251.11 \mathrm{de}$ & $224.38 \mathrm{c}$ & $0.42 \mathrm{a}$ \\
\hline S. tomentosa (75\%) & $268.1 \mathrm{a}$ & $239.1 \mathrm{~b}$ & $1.32 b$ & $1.41 \mathrm{C}$ & $1.11 \mathrm{a}$ & $0.36 a$ & 255.75 de & $226.19 \mathrm{c}$ & $0.36 \mathrm{c}$ \\
\hline S. tomentosa (50 \%) & $82.8 \mathrm{~cd}$ & $62.8 \mathrm{de}$ & $0.32 \mathrm{e}$ & $0.21 \mathrm{de}$ & $0.32 \mathrm{~cd}$ & $0.25 \mathrm{bcd}$ & $245.71 \mathrm{e}$ & $225.0 \mathrm{c}$ & $0.39 b$ \\
\hline S. tomentosa (25\%) & $112.6 \mathrm{c}$ & $76.2 d$ & $0.57 \mathrm{~d}$ & $0.53 d$ & $0.82 \mathrm{~b}$ & $0.34 a$ & $195.11 \mathrm{f}$ & $166.48 d$ & $0.30 \mathrm{~d}$ \\
\hline S. tomentosa (0 \%) & $39.2 \mathrm{e}$ & $33.8 \mathrm{e}$ & $0.18 \mathrm{e}$ & $0.11 \mathrm{e}$ & $0.25 d$ & $0.29 \mathrm{abc}$ & $234.6 \mathrm{e}$ & $205.13 \mathrm{~cd}$ & $0.38 \mathrm{bc}$ \\
\hline
\end{tabular}

$\S$ Valores con la misma letra en cada columna no son diferentes estadísticamente (Duncan, 0.05). NH: número de hojas, AF: área foliar, PSH: peso seco de hojas, PST: peso seco de tallos, PSR: peso seco de raíz, TRC: tasa relativa de crecimiento, AFE: área foliar específica, RAF: razón de área foliar, RPF: relación peso foliar.

porte de las plantas de Stevia se modificó como respuesta a los sustratos utilizados. La variación en peso seco de los órganos refleja a nivel fisiológico los cambios bioquímicos que se manifiestan en función de la nutrición (Barraza et al., 2004). Los datos del Cuadro 3 muestran que el aumento en la cantidad de vermicompost tiene un efecto positivo sobre el crecimiento de los órganos, ya que en las dos especies de Stevia no se encontraron diferencias estadísticas en el $\mathrm{NH}$ con el 75 y $100 \%$ de vermicompost; sin embargo, la biomasa aérea en S. tomentosa aumentó con $100 \%$ de vermicompost. Los valores más altos de PST se obtuvieron en S. pilosa con 75 y $100 \%$ de vermicompost, mientras que el PSR se incrementó en ambas especies con $75 \%$ de vermicompost, lo que significa que S. pilosa y S. tomentosa prefieren desarrollarse en ambientes donde la materia orgánica ya está humificada; esto puede ser una alterna- tiva para considerar en la producción a nivel de vivero con fines de reinserción en su hábitat natural.

En la eficiencia fotosintética se observan diferencias entre tratamientos (Cuadro 3). Los valores más altos en la TRC se obtuvieron con S. tomentosa en la mayoría de los tratamientos, lo que reflejó una acumulación de carbono fijado e incorporado a la biomasa estructural de la planta. En S. pilosa, el AFE, que muestra la variación de crecimiento, fue más eficiente que en $S$. tomentosa con un valor de $397.05 \mathrm{~cm}^{2} \mathrm{~g}^{-1}$ (100\% de vermicompost). Se han encontrado correlaciones positivas entre AFE y los contenidos de N y $P$ (Wright et al., 2001). Valores altos en AFE indican menor dureza y mayor calidad nutritiva de la planta. La misma tendencia se presentó con la RAF $\left(329.12 \mathrm{~cm}^{2} \mathrm{~g}^{-1}\right)$. En contraste, S. tomentosa presentó los valores más altos de RPF, 
lo que muestra mayor dureza de la hoja. La información sobre estas dos especies de Stevia es muy escasa; sin embargo, es posible afirmar que los valores significativamente menores entre especies (caso $\mathrm{S}$. tomentosa) reflejan una mayor dureza de la hoja que puede interpretarse como una adaptación al ambiente.

Ewané et al. (2012) indican que hay una alta correlación entre los índices AFE y RAF con los componentes citoplasmáticos como proteínas, minerales y ácidos orgánicos; este fue el caso de S. pilosa. Sin embargo, valores bajos indican que existe mayor cantidad de componentes de la pared celular, especialmente lignina. En concordancia con lo anterior se pudo observar que las hojas de S. tomentosa en todas las proporciones de vermicompost fueron más duras y quebradizas durante el ciclo del cultivo.

\section{Componente del crecimiento}

El NH varió en función del tiempo, la especie y proporciones de sustrato. En el último muestreo, S. tomentosa produjo más hojas que S. pilosa con 75 y $100 \%$ de vermicompost (Figura 1).

El AF se incrementó en función del tiempo y las proporciones de vermicompost. A los 82 dde las hojas de $\mathrm{S}$. tomentosa produjeron la mayor cantidad de AF con las mezclas 25:75 y 0:100 (fibra de coco:vermicompost) con valores de $440.87 \mathrm{~cm}^{2}$ y $426.98 \mathrm{~cm}^{2}$, respectivamente (Figura 2). La misma tendencia se presentó en S. pilosa.

Las combinaciones de los sustratos permitieron diferenciar la cantidad producida en biomasa aérea en ambas especies. A los 82 dde S. tomentosa tuvo valores significativos en la acumulación de materia seca en hojas, lo que supera a S. pilosa con las proporciones 75 y $100 \%$ de vermicompost (Figura 3). Aunque el PST se incrementó en ambas especies en las mismas proporciones, S. tomentosa presentó tallos más leñosos que S. pilosa. En ambas especies el desarrollo de raíz se modificó en función de la cantidad de vermicompost (Figura 3), pues con $100 \%$ las raíces fueron abundantes pero delgadas, mientras que con el $25 \%$ el número de raíces fue menor pero éstas fueron más gruesas. Estos resultados denotan el efecto positivo del humus sobre el desarrollo de las raíces de las plantas, debido a la presencia de compuestos promotores del crecimiento y actividad microbiana que favorece el desarrollo de la raíz (Marschner, 2012). Se especula que la presencia de sustancias similares a fitohormonas en el humus de lombriz (Arancon et al., 2010) ocasionó la respuesta observada, tanto en crecimiento como en el desarrollo de las especies de Stevia.

\section{Eficiencia fotosintética}

En ambas especies la TRC fue máxima en el primer muestreo (de 42 a 50 dde). En S. pilosa la TRC fue mayor con la adición de 50, 75 y $100 \%$ de vermicompost (0.954 $\mathrm{g} \mathrm{g}^{-1} \mathrm{~d}^{-1}, 0.893 \mathrm{~g} \mathrm{~g}^{-1} \mathrm{~d}^{-1}$ y $0.838 \mathrm{~g} \mathrm{~g}^{-1} \mathrm{~d}^{-1}$, respectivamente), lo que supera entre 27 y $45 \%$ a S. tomentosa con el 25 $\%$ de vermicompost (0.657 $\mathrm{g} \mathrm{g}^{-1} \mathrm{~d}^{-1}$ ) (Figura 4). La planta en la etapa vegetativa es más eficiente para producir tejido, porque depende de la fotosíntesis total y la respiración (Pommerening y Muszta, 2016), mientras que durante la senescencia la planta disminuye su actividad meristemática y elongación celular (Muhammad et al., 2014). Desde el punto de vista de las relaciones entre los órganos que producen fotoasimilados y los que los consumen, la TRC es un indicador para medir esa eficiencia (Nassi o Di Nasso et al., 2011).

El AFE, que representa la relación entre el espesor y la superficie de la hoja (Clavijo, 1989), y en forma indirecta el efecto de la nutrición del cultivo y de la estructura del dosel, se incrementó en las primeras etapas de desarrollo en ambas especies. En S. pilosa el valor fue $62.25 \%$ más que en $\mathrm{S}$. tomentosa, independientemente de las relaciones de vermicompost (Figura 5). En ambas especies los valores de AFE fueron decreciendo en función del tiempo, debido a que los fotoasimilados se distribuyeron a las zonas de división y expansión celular de mayor demanda (meristemos). Las especies con crecimiento rápido bajo óptimas condiciones son aquellas que tienen la mayor AFE (Poorter y De Jong, 1999), como es el caso de S. pilosa con $100 \%$ de vermicompost.

La RAF es la relación entre el área foliar de la planta y la materia seca total (Clavijo, 1989), índice que muestra la proporción de asimilados fotosintéticos que son asignados a la producción de follaje. En la presente investigación, a los 42 dde S. pilosa, con 50, 75 y $100 \%$ de vermicompost obtuvo los valores más altos en RAF con respecto a $S$. tomentosa (Figura 6). En muestreos posteriores del cultivo este índice fue decreciendo y mostró estabilidad.

\section{Masa foliar}

El índice de la RPF indica el peso seco foliar con respecto al peso seco total (Clavijo, 1989) y es un indicador de la masa foliar de la planta que expresa cuándo y cuántos fotoasimilados se traslocan en función del tiempo. Los valores obtenidos para este factor a los 42 dde muestran que $S$. tomentosa asignó una mayor cantidad de fotoasimilados al follaje con 0 y $25 \%$ de vermicompost en comparación con S. pilosa (Figura 7). Por lo tanto, a los 42 dde $S$. tomentosa invirtió más tejido foliar en 0 y $25 \%$ de vermicompost debido a la falta de nutrimentos, para poder 
FC-VC

- 0-100

- 25-75

$\Delta 50-50$

$* 75-25$

$\bullet 100-0$

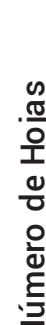

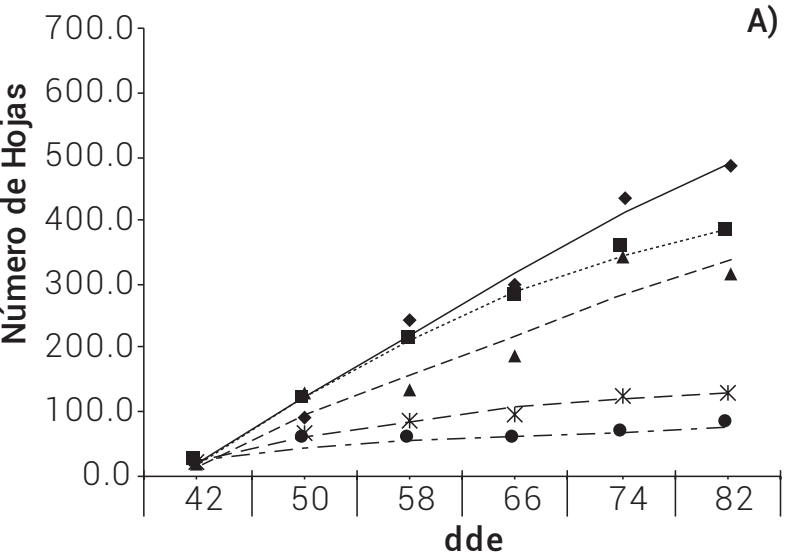

FC-VC

- 0-100- $y=1.8 \mathrm{E}+01+2.8 \mathrm{E}-01 \mathrm{x}+3.5 \mathrm{E}-01 \mathrm{x}^{2} \mathrm{R}^{2}=0.99$

- 25-75 …... $y=-1.7 E+01+1.1 E+01 x+3.8 E-02 x^{2} R^{2}=0.98$

$\triangle 50-50---y=2.5 E+01-6.4 E-01 x+1.0 E-01 x^{2} R^{2}=0.96$

$* 75-25---y=1.3 E+00+6.4 E+00 x-5.8 E-02 x^{2} R^{2}=0.97$

- $100-0--y=1.7 E+01+1.6 E+01 x+2.6 E-02 x^{2} R^{2}=0.94$

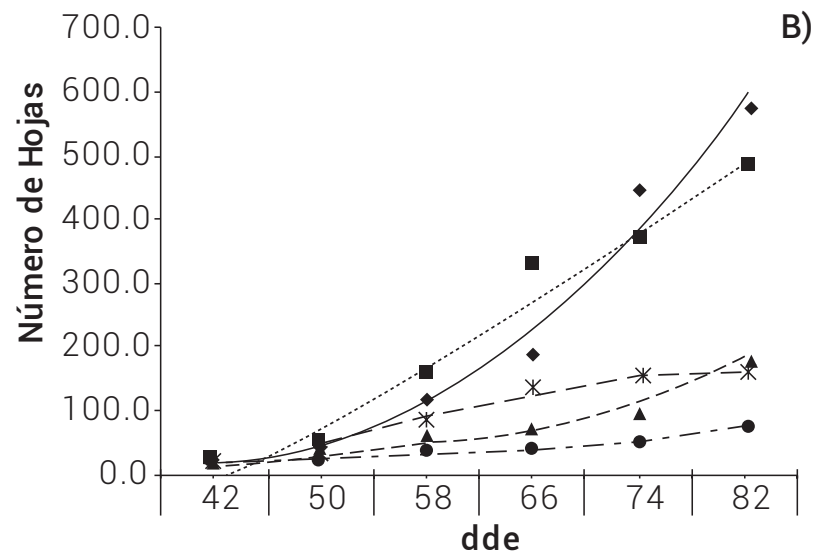

Figura 1. Dinámica del número de hojas en plantas de S. pilosa (A) y S. tomentosa (B), en función de la proporción fibra de coco:vermicompost y el tiempo de muestreo, FC-VC: fibra de coco-vermicompost, dde: días despues de la emergencia.

FC-VC

- $0-100-y=2.5 E+01+1.3 E+01 x-8.2 E-02 x^{2} R^{2}=0.99$

- 25-75 …... $y=3.6 \mathrm{E}+01+8.9 \mathrm{E}+00 \mathrm{x}-2.2 \mathrm{E}-02 \mathrm{x}^{2} \quad \mathrm{R}^{2}=0.99$

$\Delta 50-50---y=4.3 E+01+3.7 E+00 x+4.4 E-02 x^{2} R^{2}=0.96$

$* 75-25---y=2.8 E+01+2.3 E+00 x-2.8 E-02 x^{2} R^{2}=0.98$

- $100-0--\mathrm{y}=2.5 \mathrm{E}+01+8.9 \mathrm{E}-01 \mathrm{x}-6.7 \mathrm{E}-03 \mathrm{x}^{2} \mathrm{R}^{2}=0.99$

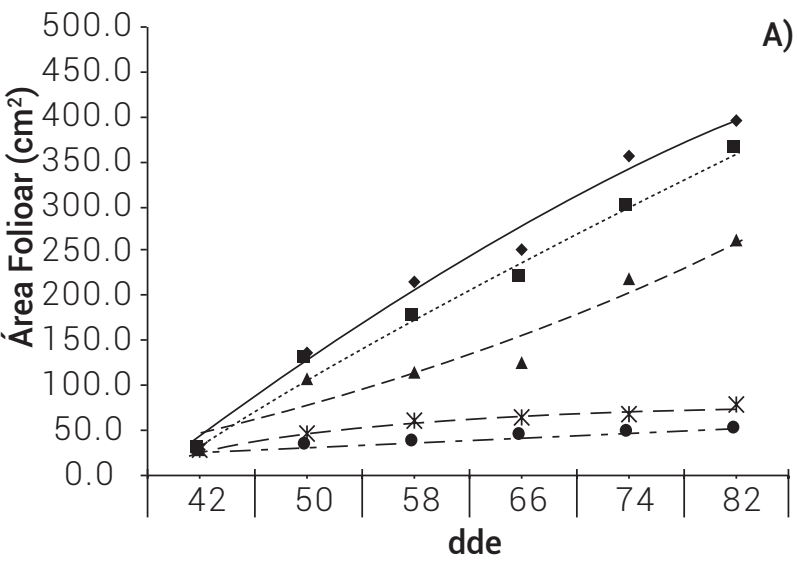

FC-VC

- $0-100-y=-5.5 E+01+7.2 E+00 x+1.0 E-01 x^{2} R^{2}=0.99$

- 25-75 …... $y=-1.3 \mathrm{E}+01+9.5 \mathrm{E}+00 \mathrm{x}+4.1 \mathrm{E}-02 \mathrm{x}^{2} \mathrm{R}^{2}=0.99$

$\Delta 50-50---y=1.9 E+01-8.6 E-01 x+9.0 E-02 x^{2} R^{2}=0.95$

$* 75-25---y=3.2 E+00+2.6 E+00 x+1.5 E-02 x^{2} R^{2}=0.99$

- $100-0--y=1.3 \mathrm{E}+01-4.3 \mathrm{E}-01 \mathrm{x}+4.4 \mathrm{E}-02 \mathrm{x}^{2} \mathrm{R}^{2}=0.95$

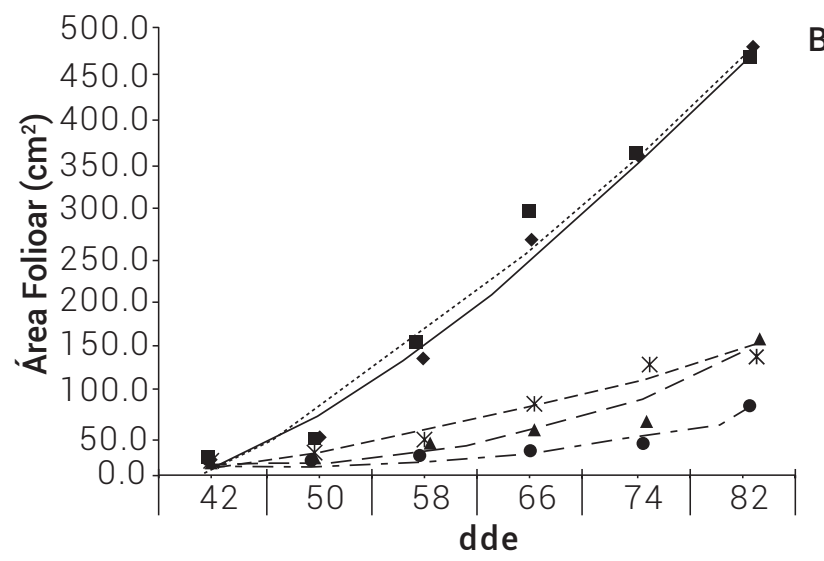

Figura 2. Dinámica del área foliar en plantas de S. pilosa (A) y S. tomentosa (B), en función de la proporción fibra de coco:vermicompost y el tiempo de muestreo, FC-VC: fibra de coco-vermicompost, dde: días despues de la emergencia. 
FC-VC

- $0-100-y=4.6 \mathrm{E}-02+2.9 \mathrm{E}-02 \mathrm{x}+3.1 \mathrm{E}-04 \mathrm{x}^{2} \mathrm{R}^{2}=0.99$

$\Delta 50-50---y=6.6 E-02+2.3 E-02 x+2.5 E-04 x^{2} \quad R^{2}=0.99$

$* 75-25--y=2.6 \mathrm{E}-02+1.9 \mathrm{E}-02 x-1.8 \mathrm{E}-04 \mathrm{x}^{2} \mathrm{R}^{2}=0.99$

- $100-0--y=4.9 E-02+5.9 E-03 x-3.1 E-05 x^{2} R^{2}=0.96$

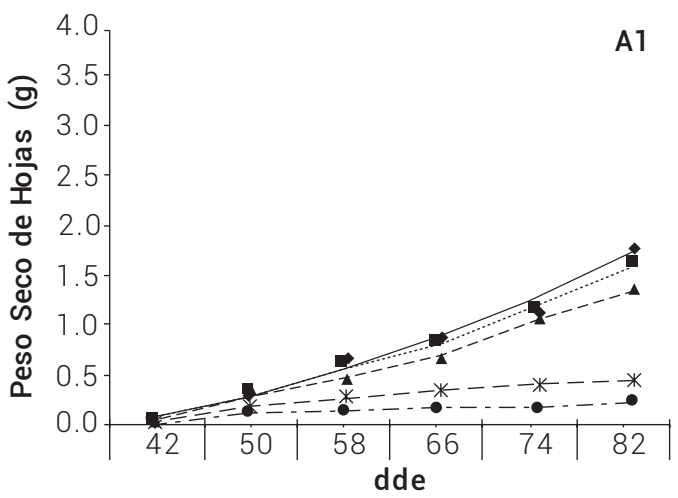

FC-VC

- $0-100-y=1.8 E-01-2.8 E-02 x+3.6 E-03 x^{2} R^{2}=0.99$

- 25-75 …... $y=1.4 E-01+1.9 E-02 x+1.8 E-03 x^{2} R^{2}=0.99$

$\Delta 50-50---y=7.8 E-02+3.5 E-02 x+6.3 E-04 x^{2} R^{2}=0.98$

$* 75-25---y=-4.9 E-02+3.2 E-02 x-1.6 E-04 x^{2} R^{2}=0.99$

- $100-0--y=5.2 E-02-1.2 E-04 x+3.6 E-04 x^{2} R^{2}=0.99$

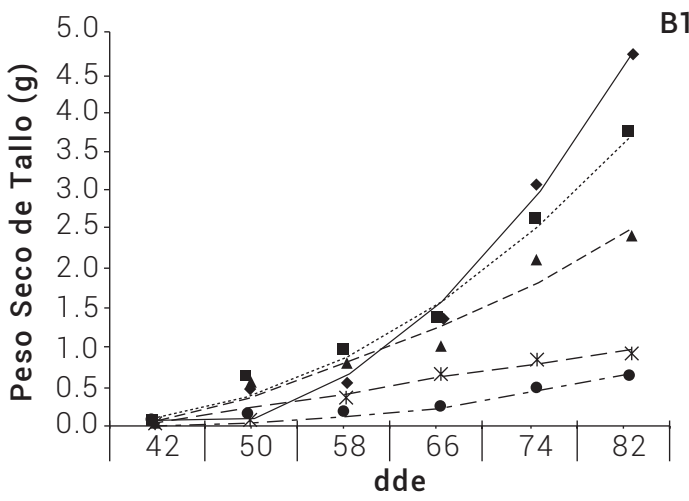

FC-VC

- $0-100-y=1.5 \mathrm{E}-01-2.1 \mathrm{E}-02 x+1.7 \mathrm{E}-03 \mathrm{x}^{2} \mathrm{R}^{2}=0.99$

- 25-75 …... $y=1.9 E-02+1.9 E-02 x+8.6 E-04 x^{2} \quad R^{2}=0.99$

$\Delta 50-50---y=-2.3 E-02+4.2 E-02 x+8.5 E-05 x^{2} R^{2}=0.99$

$* 75-25--y=3.5 E-02+2.9 E-02 x+3.3 E-05 x^{2} R^{2}=0.99$

- 100-0 - - $y=6.7 \mathrm{E}-02+8.7 \mathrm{E}-03 \mathrm{x}+1.8 \mathrm{E}-04 \mathrm{x}^{2} \mathrm{R}^{2}=0.97$

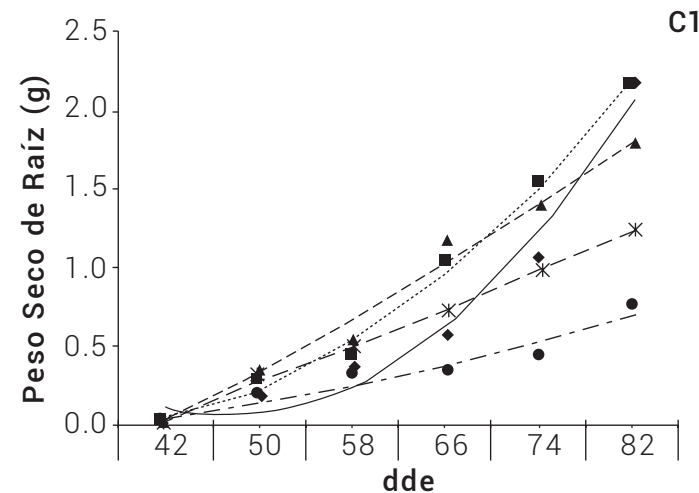

- 25-75 …... $y=5.5 E-02+2.9 E-02 x+2.3 E-04 x^{2} R^{2}=0.99$

FC-VC

- $0-100-y=-1.2 E-01+2.3 E-02 x+1.7 E-03 x^{2} R^{2}=0.96$

- 25-75 …... $y=-7.0 E-02+2.3 E-02 x+1.2 E-03 x^{2} \quad R^{2}=0.99$

4 $50-50--y=2.3 E-02+3.8 E-03 x+2.9 E-04 x^{2} R^{2}=0.99$

$* 75-25---y=-1.7 \mathrm{E}-02+1.4 \mathrm{E}-02 x+3.9 \mathrm{E}-04 \mathrm{x}^{2} \mathrm{R}^{2}=0.99$

$\bullet 100-0--y=4.8 \mathrm{E}-03+5.8 \mathrm{E}-03 \mathrm{x}+5.7 \mathrm{E}-05 \mathrm{x}^{2} \mathrm{R}^{2}=0.99$

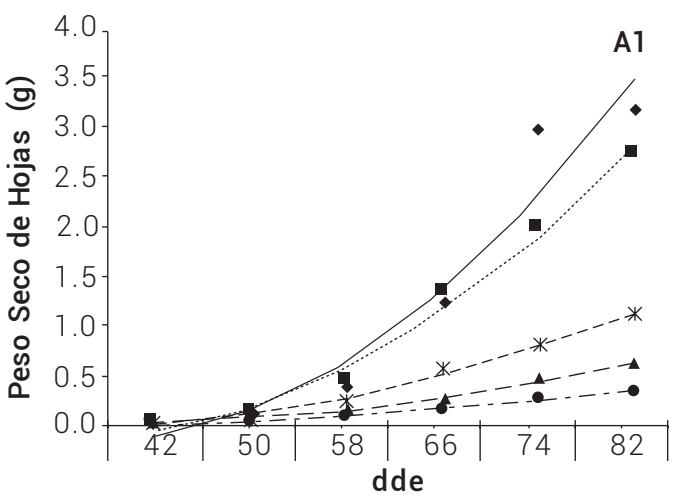

FC-VC

- $0-100-y=2.3 E-01-8.3 E-02 x+4.8 E-03 x^{2} R^{2}=0.99$

- 25-75 …... $y=8.5 E-03-8.8 E-03 x+2.3 E-03 x^{2} R^{2}=0.99$

$\Delta 50-50--y=2.7 E-02-5.5 E-04 x+2.7 E-04 x^{2} R^{2}=0.99$

$* 75-25---y=-5.8 \mathrm{E}-02+1.4 \mathrm{E}-02 x+3.8 \mathrm{E}-04 \mathrm{x}^{2} \mathrm{R}^{2}=0.98$

- $100-0--y=4.9 E-03-5.9 E-04 x+1.7 E-04 x^{2} R^{2}=0.99$

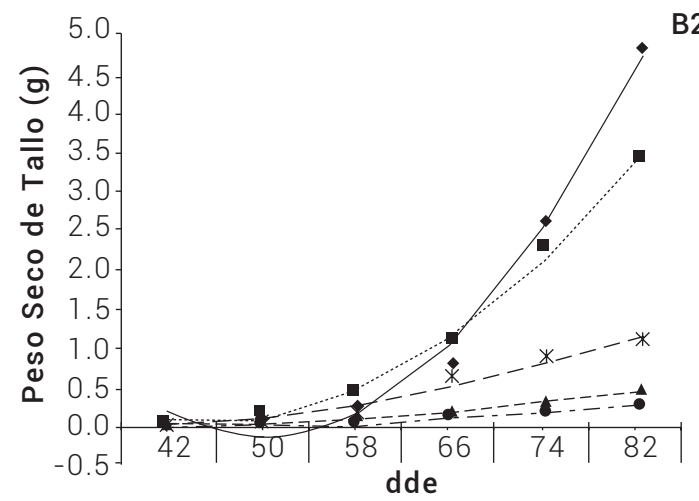

FC-VC

- $0-100-y=-3.1 E-02+7.0 E-03 x+1.1 E-03 x^{2} \quad R^{2}=0.98$

- 25-75 …... $y=-1.1 \mathrm{E}-01+3.1 \mathrm{E}-02 \mathrm{x}+7.1 \mathrm{E}-04 \mathrm{x}^{2} \mathrm{R}^{2}=0.98$

$\Delta 50-50---y=2.6 E-02-1.7 E-03 x+4.7 E-04 x^{2} \quad R^{2}=0.99$

$* 75-25---y=-2.8 E-02+2.3 E-02 x+4.1 E-04 x^{2} R^{2}=0.99$

- $100-0--y=1.7 E-02+2.6 E-04 x+3.2 E-04 x^{2} R^{2}=0.99$

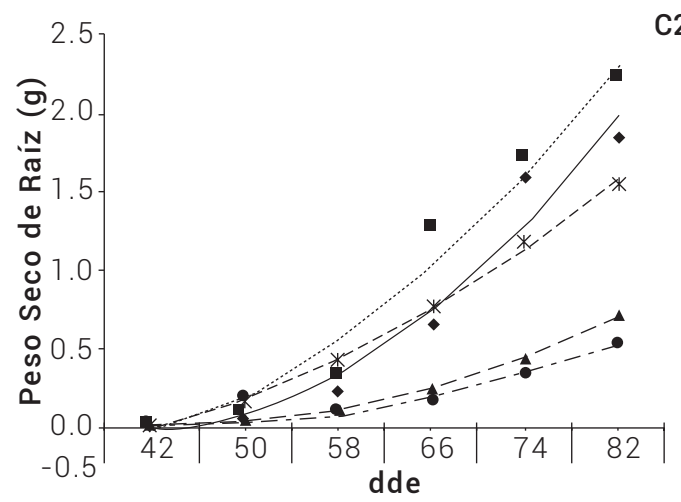

C2

Figura 3. Dinámica de pesos secos de hojas (A), tallos (B) y raíz (C) en plantas de S. pilosa (1) y S. tomentosa (2), en función de la proporción fibra de coco:vermicompost y el tiempo de muestreo, FC-VC: fibra de coco-vermicompost, dde: días despues de la emergencia. 
FC-VC

- $0-100-y=1.3 E+00-8.2 E-02 x+1.4 E-03 x^{2} \quad R^{2}=0.92$

- 25-75 …... $y=1.4 E+00-8.9 E-02 x+1.5 E-03 x^{2} R^{2}=0.92$

$\Delta 50-50--y=1.5 E+00-9.7 E-02 x+1.6 E-03 x^{2} \quad R^{2}=0.89$

$* 75-25---y=9.9 E-01-5.4 E-02 x+7.9 E-04 x^{2} R^{2}=0.99$

- 100-0 - - $y=8.6 E-01-5.7 E-02 x+9.9 E-04 x^{2} R^{2}=0.93$

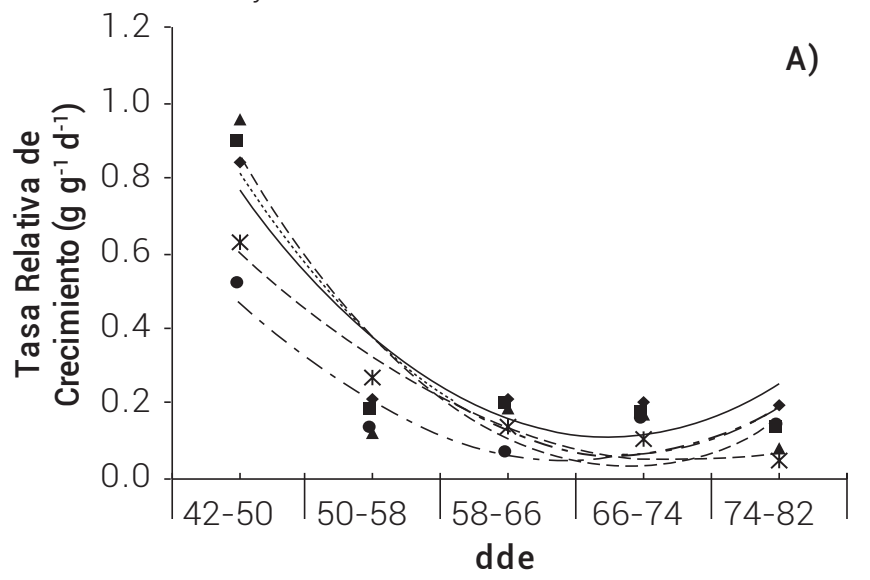

FC-VC

- $0-100-y=1.2 E-01+4.9 E-03 x-1.7 E-04 x^{2} R^{2}=0.97$

- 25-75 …... $y=2.1 E-01-1.6 E-03 x-7.0 E-05 x^{2} \quad R^{2}=0.97$

$\Delta 50-50$ - - $y=1.5 E-01-4.0 E-03 x+3.8 E-05 x^{2} R^{2}=0.99$

$* 75-25--y=2.9 E-01-9.5 E-03 x+7.6 E-05 x^{2} R^{2}=0.99$

- 100-0 - - $y=2.1 E-01-6.9 E-03 x+7.0 E-05 x^{2} R^{2}=0.99$

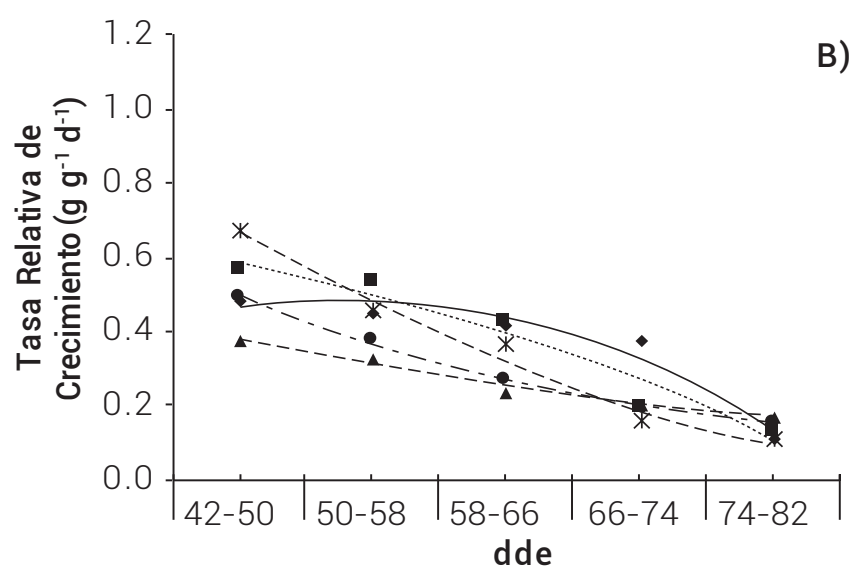

Figura 4. Dinámica de la tasa relativa de crecimiento en plantas de S. pilosa (A) y S. tomentosa (B), en función de la proporción fibra de coco:vermicompost y el tiempo de muestreo. FC-VC: fibra de coco-vermicompost, dde: días despues de la emergencia.
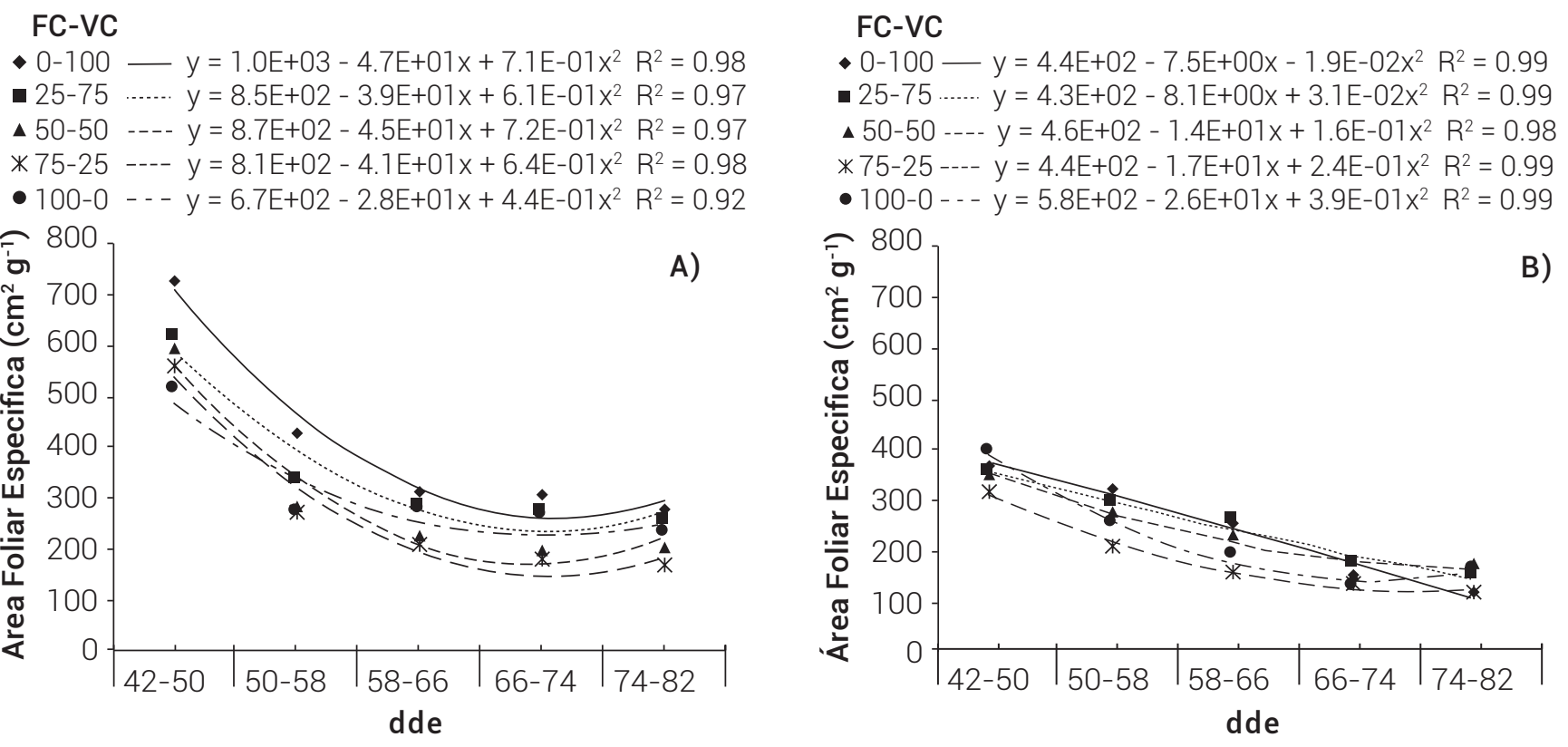

Figura 5. Dinámica del área foliar específica en plantas de S. pilosa (A) y S. tomentosa (B), en función de la proporción fibra de coco:vermicompost y el tiempo de muestreo, FC-VC: fibra de coco-vermicompost, dde: días despues de la emergencia. 
FC-VC

- 0-100-y - $8.9 \mathrm{E}+02-4.4 \mathrm{E}+01 \mathrm{x}+7.2 \mathrm{E}-01 \mathrm{x}^{2} \mathrm{R}^{2}=0.97$

- 25-75 …... $y=7.9 E+02-4.2 E+01 x+7.3 E-01 x^{2} R^{2}=0.94$

$\Delta 50-50--y=8.1 E+02-5.0 E+01 x+9.0 E-01 x^{2} R^{2}=0.93$

$* 75-25---y=7.5 \mathrm{E}+02-4.5 \mathrm{E}+01 x+7.8 \mathrm{E}-01 x^{2} \mathrm{R}^{2}=0.96$

- $100-0--y=6.7 E+02-3.4 E+01 x+6.0 E-01 x^{2} R^{2}=0.88$

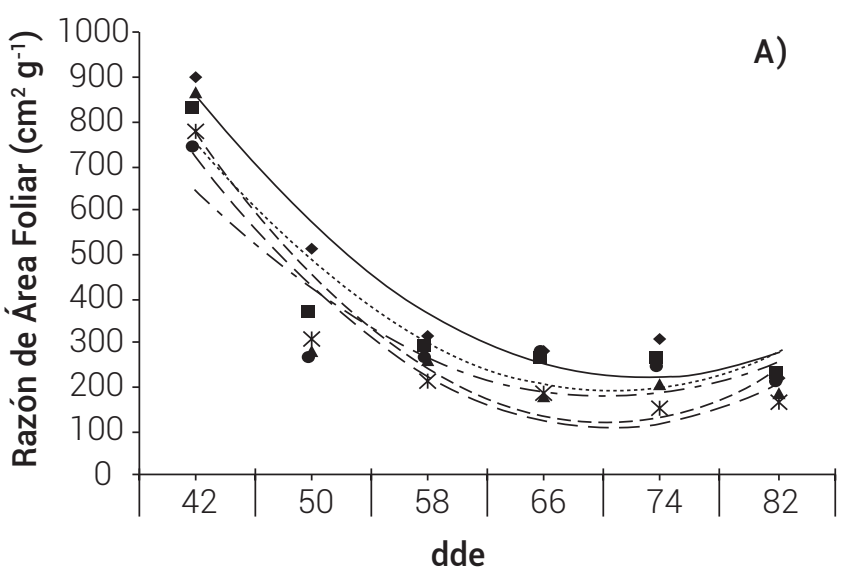

FC-VC

- $0-100-y=4.3 E+02-1.0 E+01 x+6.4 E-02 x^{2} R^{2}=0.97$

- $25-75 \ldots . . . y=4.4 E+02-1.3 E+01 x+1.4 E-01 x^{2} R^{2}=0.94$

$\Delta 50-50---y=4.4 E+02-1.8 E+01 x+2.9 E-01 x^{2} R^{2}=0.93$

$* 75-25---\mathrm{y}=4.0 \mathrm{E}+02-1.7 \mathrm{E}+01 \mathrm{x}+2.6 \mathrm{E}-01 \mathrm{x}^{2} \mathrm{R}^{2}=0.99$

- 100-0 - - $y=5.3 E+02-2.9 E+01 x+5.2 E-01 x^{2} R^{2}=0.98$

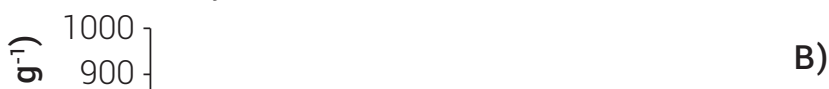

का 900

B)

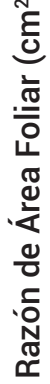

Figura 6. Dinámica de la razón de área foliar en plantas de S. pilosa (A) y S. tomentosa (B), en función de la proporción fibra de coco:vermicompost y el tiempo de muestreo, FC-VC: fibra de coco-vermicompost, dde: días despues de la emergencia.

FC-VC

- $0-100-y=3.3 \mathrm{E}-01+4.9 \mathrm{E}-03 \mathrm{x}-2.2 \mathrm{E}-04 \mathrm{x}^{2} \mathrm{R}^{2}=0.86$

- $25-75 \ldots . . . . \quad y=3.4 E-01-3.4 E-03 x+6.0 E-06 x^{2} R^{2}=0.94$

$\Delta 50-50--y=3.5 E-01-7.4 E-03 x+1.3 E-04 x^{2} R^{2}=0.99$

$* 75-25--y=3.5 \mathrm{E}-01-8.3 \mathrm{E}-03 \mathrm{x}+1.0 \mathrm{E}-04 \mathrm{x}^{2} \mathrm{R}^{2}=0.99$

- $100-0--y=3.4 E-01-7.0 E-03 x+6.1 E-05 x^{2} R^{2}=0.99$

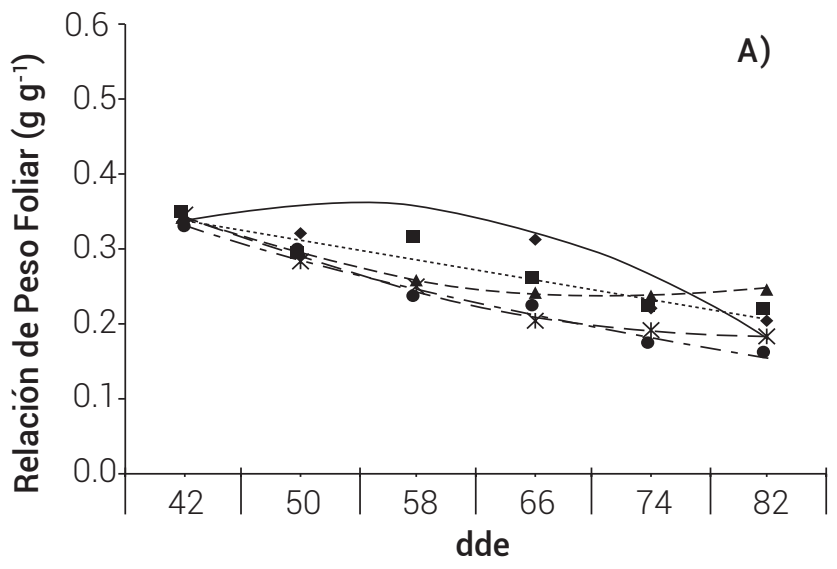

FC-VC

- $0-100-y=4.1 \mathrm{E}-01+7.2 \mathrm{E}-03 \mathrm{x}-2.2 \mathrm{E}-04 \mathrm{x}^{2} \mathrm{R}^{2}=0.97$

- $25-75 \ldots . . . . y=4.8 E-01-8.7 E-03 x+1.3 E-04 x^{2} R^{2}=0.98$

$\Delta 50-50--y=4.6 E-01-2.9 E-03 x+1.1 E-05 x^{2} R^{2}=0.96$

$* 75-25---y=5.4 E-01-2.2 E-02 x+4.1 E-04 x^{2} R^{2}=0.92$

- $100-0--y=5.5 E-01-9.4 E-03 x+8.0 E-05 x^{2} R^{2}=0.97$

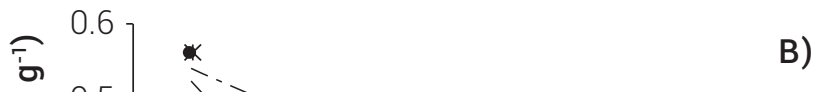

B)

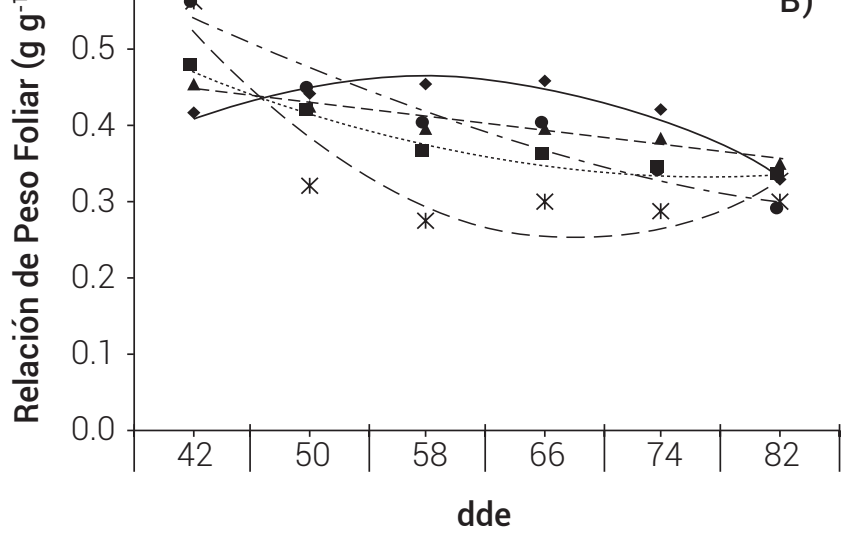

Figura 7. Dinámica de la relación de peso foliar en plantas de S. pilosa (A) y S. tomentosa (B), en función de la proporción fibra de coco:vermicompost y el tiempo de muestreo, FC-VC: fibra de coco-vermicompost, dde: días despues de la emergencia. 
captar la mayor cantidad de luz y dióxido de carbono.

Conforme avanzó el ciclo del cultivo las dos especies de Stevia disminuyeron los valores del AFE, RAF y RPF, debido a que en las primeras etapas de crecimiento la mayor parte de fotoasimilados se asignó al establecimiento del aparato foliar, cantidad que va reduciendo gradualmente a medida que la planta acumula una mayor cantidad de carbohidratos en otros órganos. Por otro lado, la disponibilidad de los nutrimentos y el desarrollo ontogénico influyó en la asignación de biomasa a los diferentes órganos de la planta, como se observa en las figuras.

\section{CONCLUSIONES}

A mayor proporción de vermicompost se observó un mayor efecto benéfico sobre el crecimiento y desarrollo de ambas especies de Stevia. Las plantas de S. pilosa crecieron mejor con las mayores concentraciones de vermicompost (75 y $100 \%$ ), debido a la considerable disponibilidad nutrimental; sin embargo, S. tomentosa creció con la menor cantidad de vermicompost ( $25 \%$ ) lo que indica que es una especie que demanda menos nutrimentos.

\section{BIBLIOGRAFÍA}

Afifi A., S. May and V. A. Clark (2012) Practical Multivariate Analysis. 5th edition. CRC Press, Taylor and Francis Group. Boca Raton, Florida, USA. $517 \mathrm{p}$.

Arancon N., C. A. Edwards, K. A. Webster and J. C. Buckerfield (2010) The potential of vermicomposts as plant growth media for greenhouse crop production. In: Vermiculture Technology: Earthworms, Organic Wastes and Environmental Management. C. A. Edwards, N. Q. Arancon and R. L. Sherman (eds.). CRC Press. Boca Raton, Florida, USA. pp:103-124.

Azarpour E., M. Moraditochaee and H. R. Bozorgi (2014) Effect of nitrogen fertilizer management on growth analysis of rice cultivars. International Journal of Biosciences 4:35-47.

Barraza F. V., G. Fischer y C. E. Cardona (2004) Estudio del proceso de crecimiento del cultivo del tomate (Lycopersicon esculentum Mill.) en el valle del Sinú medio, Colombia. Agronomía Colombiana 22:81-90.

Calderón G. y J. Rzedowski (2005) Flora Fanerogámica del Valle de México. Instituto de Ecología A.C. y Comisión Nacional para el Conocimiento y Uso de la Biodiversidad. Pátzcuaro, Michoacán. $1406 \mathrm{p}$.

Cerda-García-Rojas C. M. and R. Pereda-Miranda (2001) The phytochemistry of Stevia: a general survey. In: Stevia. The Genus Stevia. A. D. Kinghorn (ed.). Taylor \& Francis. London. pp:86-118.

Clavijo J. (1989) Análisis de crecimiento en malezas. Revista Comalfi 15:12-16.
Ewané C. A., P. Lepoivre, L. de Lapeyre de Bellaire and L. Lassois (2012) Involvement of phenolic compounds in the susceptibility of bananas to crown rot. A review. Biotechnologie, Agronomie, Société et Environnement 16:393-404

Hernández L. R., C. A. N. Catalán and P. Joseph-Nathan (1998) The chemistry of the genus Stevia (Asteraceae). Revista de la Academia Colombiana de Ciencias Exactas, Físicas y Naturales 22:229279.

Hunt R. (1982) Plant Growth Curves-The Functional Approach to Plant Growth Analysis. Edward Arnold Ltd. London. 248 p.

Hunt R. (2017) Growth analysis, individual plants. In: Encyclopedia of Applied Plant Sciences. $2^{\text {nd }}$ ed. B. Thomas, B. G. Murray and D. J. Murphy (eds.). Academic Press. Oxford, UK. pp:421-429.

Marschner P. (2012) Rhizosphere biology. In: Marschner's Mineral Nutrition of Higher Plants. 3rd edition. Marschner (ed.). Academic Press. London. pp:369-388.

Molina-Mendoza J. L., R. Galván-Villanueva, A. Patiño-Siciliano y R. Fernández-Nava (2012) Plantas medicinales y listado florístico preliminar del municipio de Huasca de Ocampo, Hidalgo, México. Polibotánica 34:259-291.

Muhammad A., S. K. Khalil, A. Zaman, Habibullah and M. S. Khan (2014) Growth analysis of soybean landraces under extended planting dates. Sarhad Journal of Agriculture 30:297-304.

Nassi o Di Nasso N., N. Roncucci, F. Triana, C. Tozzini and E. Bonari (2011) Productivity of giant reed (Arundo donax L.) and miscanthus (Miscanthus $\times$ giganteus Greef et Deuter) as energy crops: growth analysis. Italian Journal of Agronomy 6:141-147.

Pommerening A. and A. Muszta (2016) Relative plant growth revisited: towards a mathematical standarization of separate approaches. Ecological Modelling 320:383-392.

Poorter H. and R. De Jong (1999) A comparison of specific leaf area, chemical composition and leaf construction costs of field plants from 15 habitats differing in productivity. New Phytologist 143:163-176.

Prusinkiewicz P. (2004) Modeling plant growth and development. Current Opinion in Plant Biology 7:79-83.

Rincón G. N., M. A. Olarte Q. y J. C. Pérez N. (2012) Determinación del área foliar en fotografías tomadas con una cámara web, un teléfono celular o una cámara semiprofesional. Revista Facultad Nacional de Agronomía 65:6399-6405.

Sagakuchi M. and T. Kan (1982) As pesquisas japonesas com Stevia rabaudiana (Bert.) Bertoni e o esteviosideo. Ciencia e Cultura 34:235-248.

SAS, Intitute (2015) SAS/STAT user's guide Release 9.4. SAS Institute, Inc. Cary, NC, USA. 440p.

Siswadi, A. Muslim and T. Bakhtiar (2012) Variable selection using principal component and procrustes analyses and its application in educational data. Journal of Asian Scientific Research 2:856865.

Steiner A. A. (1984) The universal nutrient solution. In: Proceedings of the Sixth International Congress on Soilles Culture. Lunteren, 29 April-5 May. International Society for Soilless Culture: Secretariat of ISOSC. Wageningen, The Netherlands. pp: 633-650.

Villavicencio N. M. A. y E. B. Pérez E. (1995) Plantas Útiles del Estado de Hidalgo. Vol. 2. Universidad Autónoma del Estado de Hidalgo. Pachuca, Hidalgo. 128 p.

Wright I. J., P. B. Reich and M. Westoby (2001) Strategy shifts in leaf physiology, structure and nutrient content between species of highand low-rainfall and high-and low-nutrient habitats. Functional Ecology 15:423-434. 